\title{
Doppler Lidar Sensor for Precision Landing on the Moon and Mars
}

\author{
Farzin Amzajerdian, Larry Petway, Glenn Hines, and \\ Bruce Barnes \\ NASA Langley Research Center, MS 468, \\ Hampton, VA 23681, USA \\ 1-757-864-1533 \\ f.amzajerdian@nasa.gov
}

\author{
Diego Pierrottet, and George Lockard \\ Coherent Applications, Inc. \\ 20 Research Drive, Suite 500 \\ Hampton, VA 23666, USA \\ 1-7570864-1636 \\ diego.f.pierrottet@nasa.gov
}

\begin{abstract}
Landing mission concepts that are being developed for exploration of planetary bodies are increasingly ambitious in their implementations and objectives. Most of these missions require accurate position and velocity data during their descent phase in order to ensure safe soft landing at the pre-designated sites. To address this need, a Doppler lidar is being developed by NASA under the Autonomous Landing and Hazard Avoidance (ALHAT) project. This lidar sensor is a versatile instrument capable of providing precision velocity vectors, vehicle ground relative altitude, and attitude. The capabilities of this advanced technology have been demonstrated through two helicopter flight test campaigns conducted over a vegetation-free terrain in 2008 and 2010. Presently, a prototype version of this sensor is being assembled for integration into a rocket-powered terrestrial free-flyer vehicle. Operating in a closed loop with vehicle's guidance and navigation system, the viability of this advanced sensor for future landing missions will be demonstrated through a series of flight tests in 2012.
\end{abstract}

\section{TABLE OF ConTENTS}

1. INTRODUCTIONERROR! BOOKMARK NOT DEFINED. 2. Principal of OperationERror! BoOKMARK NOT 3. SYSTEM DESCRIPTION .3 4. SigNAL PROCESSING ..........................................3 5. HELICOPTER FLIGHT TESTS .............................4 6. SUMMARY ....................................................5 REFERENCES...................................................5

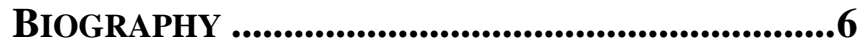

\section{INTRODUCTION}

Future robotic and manned exploration missions to the Moon, Mars, and other planetary bodies demand accurate knowledge of ground relative velocity and altitude in order to ensure soft landing at the designated landing site [1,2]. Some missions may even require landing within a few meters of pre-deployed assets or landing in a small area surrounded by rocks, craters, or steep slopes [3-5]. To meet this requirement, a Doppler lidar is being developed by
NASA under the Autonomous Landing and Hazard Avoidance (ALHAT) project [5,6]. The range and velocity measurements provided by this lidar sensor will be used by an autonomous Guidance, Navigation, and Control (GN\&C) system to accurately navigate the vehicle to the designated landing location.

The Doppler lidar will begin its operation during the powered descent phase from an altitude of a few kilometers above the ground. The GN\&C system processes the lidar data to improve position and attitude data from the Inertial Measurement Unit (IMU). The improved position and attitude knowledge along with the lidar precision vector velocity data enable the GN\&C system to continuously update the vehicle trajectory toward the landing site. In addition to the precision trajectory determination, the lidar data will play important role in performing the soft landing maneuver. For example, large robotic or manned vehicles must control their horizontal and vertical velocities to better than $0.5 \mathrm{~m} / \mathrm{s}$ in order to avoid the risk of tipping over and ensure a gentle touchdown. To control to these limits will require measurement accuracies to better than $10 \mathrm{~cm} / \mathrm{s}$. The coherent Doppler lidar, being described in this paper, exceeds these requirements by over an order of magnitude.

DEFINED

The Doppler lidar transmits three laser beams which are separated 120 degrees from each other in azimuth and are pointed 22.5 degrees from nadir. The signal from each beam provides the platform velocity and range to the ground along the laser line-of-sight (LOS). The three LOS measurements are then combined in order to determine the three components of the vehicle velocity vector, and to accurately measure altitude and attitude relative to the local ground. The LOS velocity and range precision of the lidar has been measured to be approximately $1 \mathrm{~mm} / \mathrm{sec}$ and $5 \mathrm{~cm}$ respectively. Compared with radars [2], this sensor offers several benefits including small size, high precision, high data rate, and tolerance to ground clutter.

\section{Principal of Operation}

The Doppler lidar obtains high-resolution range and velocity information from a frequency modulated continuous wave (FMCW) waveform for which the laser frequency is modulated linearly with time. Figure 1 shows the 
transmitted laser waveform and the return waveform from the target which is delayed by a time $t_{d}$, the light round trip time.

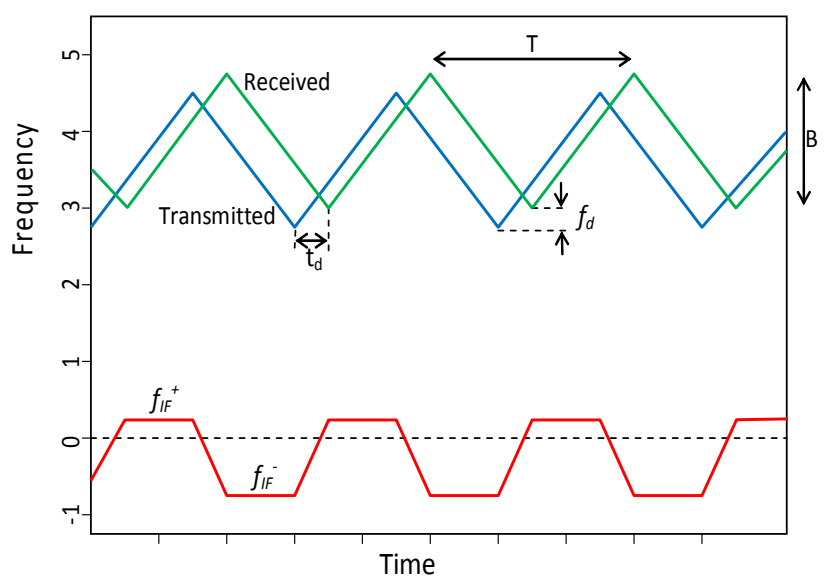

Figure 1. Laser frequency is linearly modulated to create a sawtooth waveform. Returned waveform from the target is delayed in time. In presence of platform or target velocity, the returned waveform will be Doppler shifted. The difference frequency (lower trace) obtained by homodyning the laser and returned beams contains both range and velocity information.

When mixing the two waveforms at the detector, an interference signal will be generated whose frequency is equal to the difference between the transmitted and received frequencies. This intermediate frequency (IF) is directly proportional to the target range defined by the following relationship:

$$
f_{R}=\frac{4 R B}{T c}
$$

where $\mathrm{R}$ is the range to target, $\mathrm{B}$ is the modulation bandwidth, $\mathrm{T}$ is the waveform period and $\mathrm{c}$ is the speed of light. When the target or the lidar platform is not stationary during the beam round trip time, the signal frequency will be also shifted due to the Doppler effect. Therefore by extracting the frequency during "up chirp" and "down chirp" periods of the laser waveform, both the target range and velocity can be determined. The range can be determined using Eq. (1) combined with Eq. (2):

$$
f_{R}=\frac{f_{i f}^{+}-f_{i f}^{-}}{2}
$$

The Doppler frequency shift is determined by:

$$
f_{d}=\frac{f_{i f}^{+}+f_{i f}^{-}}{2}
$$

where the Doppler frequency shift is directly related to the radial LOS velocity:

$$
f_{d}=\frac{2 V}{\lambda}
$$

To obtain horizontal and vertical velocity components, three measurements are made using three different sensor laser beams having a-priori pointing knowledge as shown in Figure 2. The platform vector velocity, $\vec{V}=v_{x} \hat{x}+v_{y} \hat{y}+$ $v_{z} \hat{z}$, is defined in a coordinate system with its origin in the lidar optical head and its axes in the platform reference frame. Then the three independent yet instantaneous measured LOS velocities $M_{A}, M_{B}$, and $M_{C}$ for channels A, B, and $C$ respectively are dot-products of the Doppler lidar pointing vectors $[S]$ and the velocity vector.

$$
\left[\begin{array}{l}
M_{A} \\
M_{B} \\
M_{C}
\end{array}\right]=[S] \cdot\left[\begin{array}{l}
v_{x} \\
v_{y} \\
v_{z}
\end{array}\right]
$$

Eq. (4) provides three equations with the three unknown velocity components that make up the velocity vector $\vec{V}$, and therefore, it can be solved simultaneously.

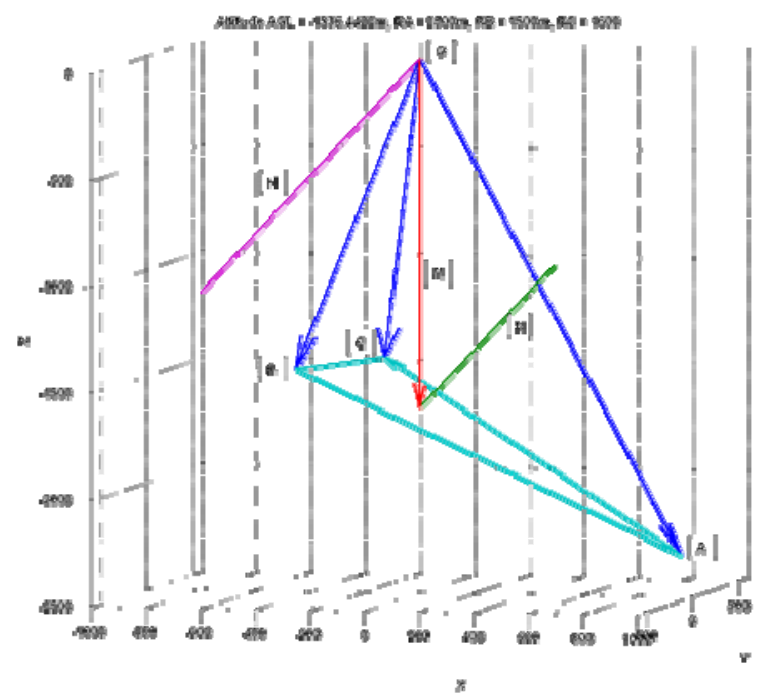

Figure 2. Doppler lidar system configuration illustrating three transmitted beams and their corresponding receivers providing line-of-sight velocity and range measurements in 3 different directions.

The vehicle altitude relative to the local ground can be obtained using either individual or all three LOS measurements. Utilizing all three LOS range measurements will provide a more representative altitude measurement as it reduces the effect of the terrain features such as boulders and is independent of the lidar nadir angle. The three LOS range measurements can also provide the vehicle attitude relative to the ground. Consider Figure 2, the vector representation of the Doppler lidar, where vectors $\vec{A}, \vec{B}$, and $\vec{C}$ are the unit vectors of channels $\mathrm{A}, \mathrm{B}$, and $\mathrm{C}$, respectively, multiplied by the measured range of each channel. $\mathrm{O}$ is a point corresponding to the origin of the Doppler lidar reference frame and 
vectors $A B, B C$ and $C A$ form the ground plane $P$ obtained by

$$
\begin{aligned}
& A B=O B-O A \\
& B C=O C-O B \\
& C A=O A-O C
\end{aligned}
$$

$N$ is the normal unit vector of the plane $P$ given by

$$
N=\frac{B C x A B}{|N|}
$$

$M$ is a median vector originating at $\mathrm{O}$ and parallel to the lidar reference frame z-axis. The magnitude of $M$ is $R_{M}$, and it is used to calculate the above ground altitude (AGL) of the vehicle ( $H$ in Fig. 2 ). Vector $M$ is defined as

$$
M=R_{M} \cdot\left[\begin{array}{c}
0 \\
0 \\
-1
\end{array}\right] \cdot\left[\begin{array}{l}
\hat{x} \\
\hat{y} \\
\hat{z}
\end{array}\right]=-R_{M} \hat{z}
$$

The median vector amplitude $R_{M}$ is found by noting that the vector difference $(M-O A)$ is a vector which lies on the ground plane $\mathrm{P}$, and therefore can be solved from:

$$
N .(M-O A)=N \cdot\left(-R_{M}-O A\right)=0 \text { (9) }
$$

Once $R_{M}$ is known, and recognizing that $H$ is parallel to $N$, the vehicle altitude is calculated from

$$
H=R_{M} \cdot\left(\frac{N \cdot M}{|N| \cdot|M|}\right)
$$

\section{SYSTEM DESCRIPTION}

Figure 3 illustrates the system design concept utilizing an optical homodyne configuration. A relatively low power, single frequency laser operating at eye safe wavelength of 1.55 micron, is used as the master oscillator. The output of this laser is modulated per the waveform of Figure 1. Part of the laser output is amplified to be transmitted and the remaining is used as the local oscillator (LO) for optical homodyne detection. The lidar transmits three laser beams which are separated 120 degrees from each other in azimuth and are pointed 22.5 degrees from nadir. The signal from each beam provides the platform velocity and range to the ground along the laser line-of-sight (LOS). The three LOS measurements are then combined in order to determine the three components of the vehicle velocity vector, and to accurately measure altitude and attitude relative to the local ground.

The main factors determining the precision of the Doppler lidar measurement are the spectral linewidth of laser, the linearity of the modulation waveform, the signal-to-noise ratio, and the signal processor frequency resolution $[7,8]$. Fortunately, the advances in single-frequency fiber laser, high quantum efficiency detector, high-speed analog electronics, and powerful processor technologies have enabled the development a system with sufficient precision and operational range for a number of applications including planetary landing.

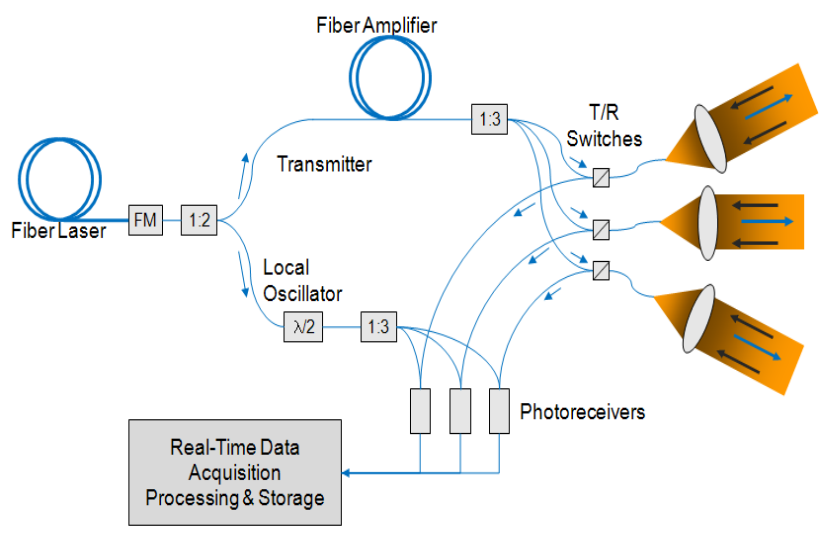

Figure 3. Doppler lidar system configuration illustrating three transmitted beams and their corresponding receivers providing line-of-sight velocity and range measurements in three different directions.

A prototype version of the Doppler lidar has recently been completed for a series of demonstration flight tests. As shown in Figure 4, the system consists of an electronics chassis and an optical head. The optical head will be mounted rigidly on the vehicle with a clear field-of-view to the ground, and connected by an armored fiber cable to the Doppler lidar electronics chassis. It is planned to demonstrate the viability of this lidar sensor system for future landing missions through a series flight tests onboard a rocket-powered terrestrial free-flyer vehicle in 2012. The test vehicle, referred to as Morpheus, is being developed by NASA-JSC to demonstrate advanced propulsion and GN\&C technologies for future landing missions [9]. The lidar will be operating in a closed-loop with a GN\&C system controlling the vehicle flight trajectory and soft landing at the selected safe site.

\section{Signal Processing}

The Doppler lidar signal processor digitizes and processes the output of three optical receivers in real-time and produces the vector velocity, altitude, and attitude data at 30 $\mathrm{Hz}$. A functional block diagram of the lidar signal processor is shown in Figure 5. The primary electronic data processing components of the Doppler lidar are a field programmable gate array (FPGA) board with a stacked analog to digital converter (ADC) daughter-board, a single board computer (SBC), and an analog and digital data acquisition (DAQ) board. The digitized outputs of the three analog receivers are demultiplexed in time to separate the up-ramp and down-ramp portions of the signal. The FPGA then applies a set of high-resolution fast Fourier transforms to the up and down-ramp segments of each of the three received signals. 

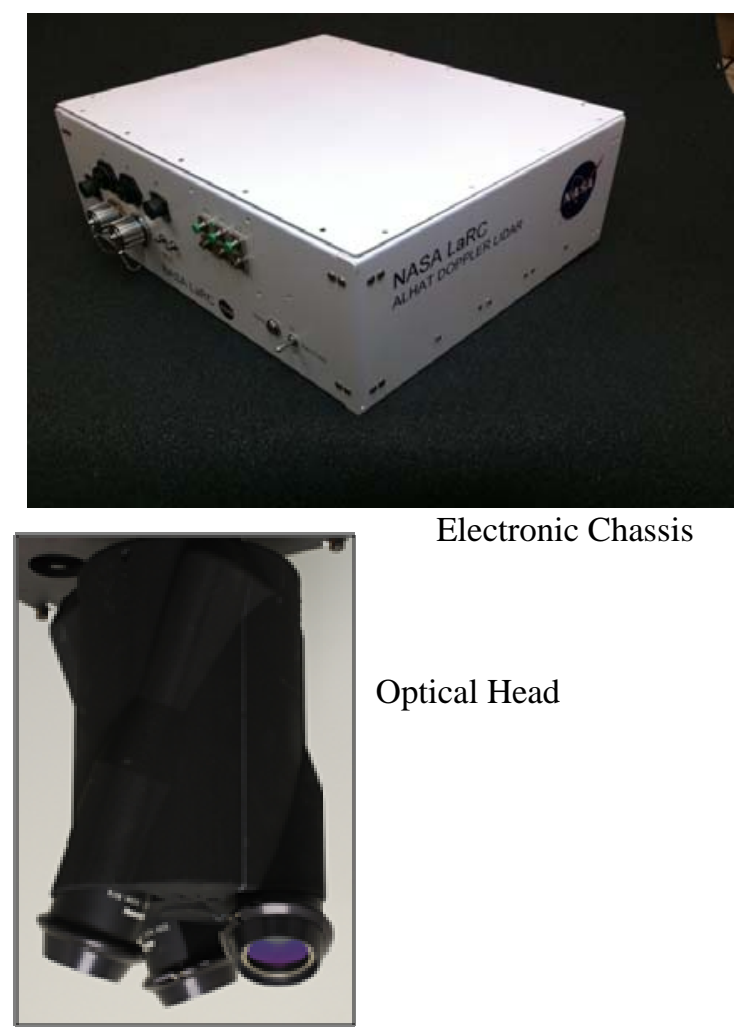

Figure 4. Prototype Doppler lidar system consisting of an electronic chassis and an optical head. All the lidar components, including transmitter laser, photoreceivers, and real-time processor, are housed in the electronic chassis. The optical head consists of three transmit and receive lenses connected to the electronic chassis by a fiber optic cable.

The FPGA firmware includes filtering and peak detection algorithms for reliable extraction of all six frequencies that in turn produce the LOS velocity and range data. The SBC performs the computations that generate the components of the vector velocity, and the vehicle altitude and attitude with respect to ground. The SBC functions as the Command and Control (C\&C) system which responds to commands from the vehicle, transmits the instrument's measurements, interfaces to the FPGA, and controls and monitors the internal components of the lidar. These boards consist of mostly commercial-off-the-shelf (COTS) components, but have a clear potential path to space qualification $[10,11]$.

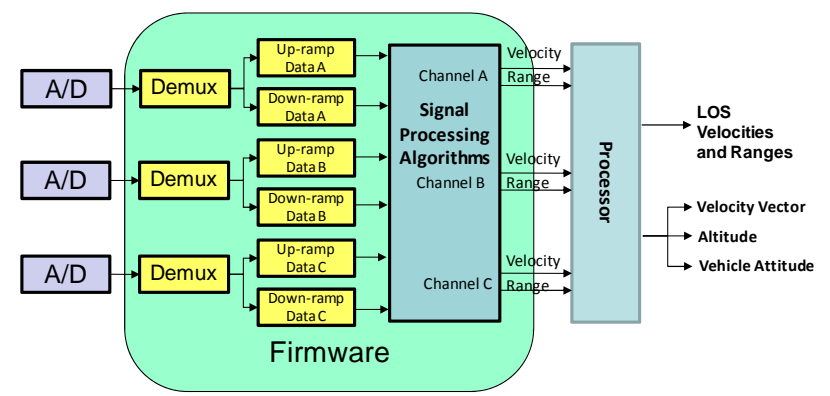

Figure 5. Real-time signal processor functional diagram.

\section{Helicopter Flight TESTS}

The capabilities of the Doppler lidar were evaluated and its performance characterized through two helicopter flight test campaigns. These campaigns were facilitated by NASAJPL, and were held at NASA-Dryden in California over vegetation-free terrain $[12,13]$. The first test was conducted in 2008 using a breadboard version of the lidar. This test consisted of several passes in figure 8 patterns over both flat and rough terrains. The lidar head was mounted in a gimbal looking nadir during each pass. The maximum altitude of this test was limited by the helicopter operational ceiling of about 1245 meters above the ground. The results indicated excellent agreement between the Doppler lidar velocity data and the numerically derived velocity from the data collected by a GPS receiver. Comparison of the Doppler lidar and GPS altitude data was somewhat difficult since GPS provides altitude data relative to the global sea level while the lidar directly measures the ground-relative altitude.

The data collected during the breadboard flight test proved to be very valuable in improving efficiency and developing the real-time signal processing algorithms, and the lessons learned help with the design of a compact and rugged prototype system. In 2010, the second generation lidar was assembled with a configuration similar to the system shown in Figure 4, except its data acquisition and processing unit was housed in a separate chassis. Characterization of this system at the Lidar Static Test Range at NASA-LaRC revealed measurement precisions of approximately 1 $\mathrm{mm} / \mathrm{sec}$ in LOS velocity and $5.0 \mathrm{~cm}$ in LOS range. A test campaign of the sensor mounted on a helicopter followed the static tests. This campaign provided the necessary data to improve the signal processor firmware and characterize the effects of laser speckle on the measurements. Laser speckle is random amplitude modulation of the return signal caused by the laser beam reflection off rough surfaces. Therefore, the signal level occasionally falls below the detection threshold resulting in a false alarm. The outliers in the data shown in Figure 6 represent the false alarms.

Figures 6 and 7 are examples of the later flight test comparing the Doppler lidar data to the data from a highgrade Inertial Measurement Unit (IMU) and GPS system built by Applanix. The Doppler lidar data shows excellent agreement with the IMU/GPS data. At the scale of these plots, the Doppler lidar and IMU/GPS data essentially overlap and are not distinguishable. The mean value of discrepancy in magnitude of the vector velocity measurements was about $3.5 \mathrm{~cm} / \mathrm{sec}$. As explained above, quantifying the altitude discrepancies between the Doppler lidar and IMU/GPS measurements is challenging since one measures altitude with respect to the ground and the other relative to sea level. For the altitude plot of Figure 6, a fixed bias was subtracted from the GPS data so it could be compared with the above ground level (AGL) altitude measurements of the lidar. This ground altitude bias was determined from the lidar data at the beginning of the flight 
taken over a flat, dry lake. The discrepancy in altitude measurements is very small and within the scale of the ground surface roughness. The analysis of the data collected from this latter helicopter flight test campaign reveals performance improvements in several aspects such as higher signal detection efficiency and lower false alarm rate. A more detailed description of the Doppler lidar and the results of the latest helicopter test flights are provided in a recent publication [13].

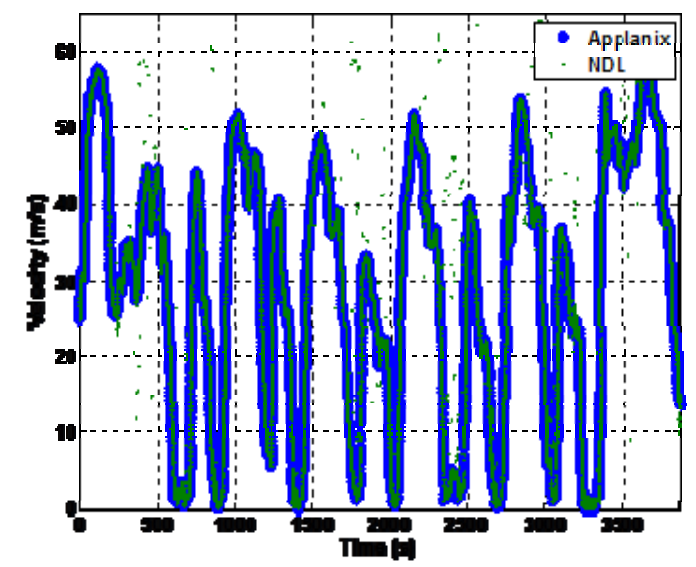

Fig. 6. Example of helicopter flight test data, comparing the magnitude of platform velocity provided by the Doppler lidar and a high grade IMU/GPS unit (Applanix).

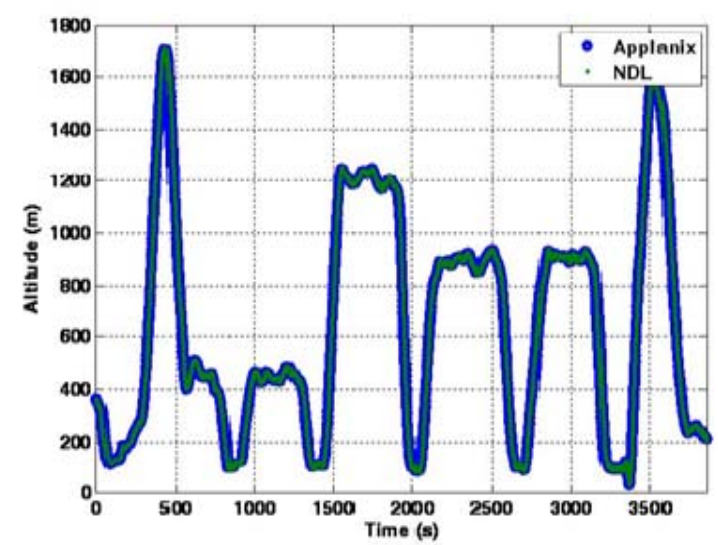

Fig. 7. AGL altitude provided by the Doppler lidar compared with Applanix IMU/GPS data corrected for ground elevation.

\section{SUMMARY}

A fiber-based coherent Doppler lidar, utilizing an FMCW waveform, has been developed and its capabilities were demonstrated through two successful helicopter flight test campaigns. This Doppler lidar is expected to play a critical role in future planetary exploration missions because of its ability to provide the necessary data for soft landing on planetary bodies and for landing missions that require precision navigation to a designated location on the ground. Compared to radars, the Doppler lidar can provide significantly higher precision velocity and altitude data at a much higher rate without concerns of measurement ambiguities or target clutter. Future work calls for testing the Doppler lidar aboard a rocket-powered free-flyer platform operating in a closed-loop configuration with the vehicle’s guidance, navigation, and control (GN\&C) unit.

\section{Acknowledgments}

The authors would like to thank the ALHAT project manager, Chirold Epp, NASA Johnson Space Center, for his support. The authors also acknowledge the ALHAT team members from the NASA Jet Propulsion Laboratory for managing the helicopter flight tests.

\section{REFERENCES}

[1] Aron A. Wolf, Jeff Tooley, Scott Ploen, Mark Ivanov, Behcet Acikmese, and Konstantin Gromov, "Performance Trades for Mars Pinpoint Landing," Proc. of IEEE Aerospace Conference, paper no.1661 (2006).

[2] Brian D. Pollard and Gregory Sadowy, "Next Generation Millimeter-wave Radar for Safe Planetary Landing,” Proc. of IEEE Aerospace Conference, paper no. 1188 (2004).

[3] Andrew E. Johnson, Allan R. Klumpp, James B. Collier, and Aron A. Wolf, "Lidar-Based Hazard Avoidance for Safe Landing on Mars,” Journal of Guidance, Control, and Dynamics, Vol. 25, No. 6 (2002).

[4] Edward C. Wong and James P. Masciarelli, "Autonomous Guidance and Control Design For Hazard Avoidance and Safe Landing on Mars,” AIAA Atmospheric Flight Mechanics Conference, Paper no. 4619 (2002).

[5] C. D. Epp, E. A. Robinson, and T. Brady, "Autonomous Landing and Hazard Avoidance Technology (ALHAT)”, Proc. of IEEE Aerospace Conference, pp.1-7 (2008).

[6] Farzin Amzajerdian, Diego Pierrottet, Larry Petway, Glenn Hines, and Vincent Roback, "Lidar systems for precision navigation and safe landing on Planetary Bodies,” Proc. of SPIE, Vol. 8192, (2011).

[7] O. Uttam, and B. Culshaw "Precision time domain reflectometry in optical fiber systems using a frequency modulated continuous wave ranging technique”, IEEE J. Lightwave Technol., LT-3, pp. 971-977 (1985).

[8] C.J. Karlsson, and F.A. Olsson "Linearization of the frequency sweep of a frequency-modulated continuous-wave semiconductor laser and the resulting 
ranging performance” App. Opt. Vol. 38, No. 15, pp 3376-3386 (1999).

[9] Project Morpheus Website

http://morpheuslander.jsc.nasa.gov/

[10] R. Hodson, K. Somervill, J. Williams, N. Bergman, and R. Jones, “An Architecture for reconfigurable computing in space,” Military and Aerospace Programmable Logic Devices (2005).

[11] K Somervill, R. Hodson, J. Williams, and R. Jones, “Reconfigurable processing module," Military and Aerospace Programmable Logic Devices (2005).

[12] D.Pierrottet, F. Amzajerdian, L. Petway, B. Barnes, and G. Lockard, "Flight test performance of a high precision navigation Doppler lidar,” Proc. SPIE, Vol. 7323 (2009).

[13] Diego Pierrottet, Farzin Amzajerdian, Larry Petway, Bruce Barnes, George Lockard, and Glenn Hines, "Navigation Doppler Lidar Sensor for Precision Altitude and Vector Velocity Measurements Flight Test Results.” Proceeding SPIE Vol. 8044, May (2011).

\section{BIOGRAPHY}

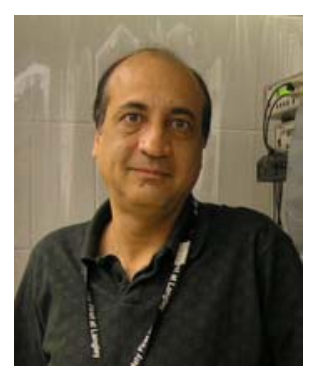

Farzin Amzajerdian received his BS and MS degrees in Electrical Engineering and BS in Mathematics from Portland State University and his PhD in Electrical Engineering from Oregon Graduate Institute. He has been working on laser remote sensing for space, military, and commercial aviation applications for the past 28 years starting with his

$\mathrm{PhD}$ thesis work and then continuing at Litton Industries and Raytheon Company. In 1993, he joined the University of Alabama in Huntsville to advance the coherent lidar technology for space applications. At UAH, he served as the lidar team lead responsible for the development of solid state lidar technologies and instruments, and as the project manager in charge of the optical system for the Space Readiness Coherent Lidar Experiment (SPARCLE) mission. He has been working at NASA Langley Research Center since 2001 leading several lidar related projects towards global measurements of the Earth and Mars atmosphere, and for aiding landing missions on planetary bodies. He is currently serving as the Instrument Lead for the Autonomous Landing and Hazard Avoidance (ALHAT) project and Principal Investigator for the Ultra-efficient Lidar Transmitter project.

Diego F. Pierrottet received his BS and MS degrees in Electrical Engineering from the University of New Mexico.
Mr. Pierrottet is the founder and owner of Coherent Applications, Inc., a company formed to provide technical services in laser radar technologies. He contributes over 18 years experience in the design, development, and evaluation of laser radar systems and laser engineering. He has fostered innovative transmitter and receiver designs in the areas of atmospheric characterization, satellite imaging, and chemical detection lidar systems. Much of his experience comes from design and field test experimentation of novel concepts as risk reduction tools for various government programs. Currently Mr. Pierrottet is a team member in the development and testing of a solid state, frequency modulated all fiber coherent Doppler Lidar and a compact long range, high precision laser altimeter Lidar system for NASA Langley Research Center, and the Autonomous Landing and Hazard Avoidance Technology for planetary landing and exploration.

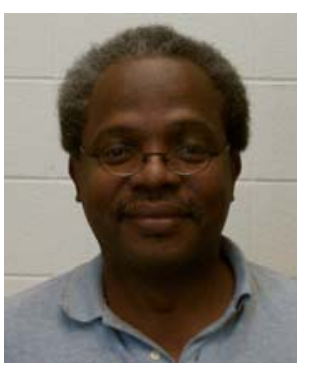

Dr. Larry Petway holds a Ph.D. in physics and has been working in the laser field for 25 years. He has been responsible for the design, development, and application of lidar systems for monitoring atmospheric species and state parameters from ground-based, airborne, and spaceborne platforms, including development of laser system electronics, detectors, optics, and signal processing. He is presently the Assistant Branch Head of the Laser Remote Sensing branch at LaRC and leading the optical design, build and demonstration of the Doppler lidar that is part of the ALHAT project. A number of innovated technological advancements were developed that has greatly improved the efficiency and signal to noise of the Doppler lidar. The first generation Doppler lidar has been built and successful field tested. The second generation design is completed incorporating improvements in a more compact configuration.

Glenn Hines holds B.S and M.S. degrees in Electrical Engineering, and M.S. and Ph.D. degrees in Computer Science. He is responsible for the design, development, and test of state-of-the-art advanced electronic systems and software for spaceflight, aviation, and atmospheric research instruments. He is a recognized engineering authority on the development and application of application specific integrated circuits (ASICs), multi-chip modules (MCMs), digital signal processors (DSPs) and field programmable gate arrays (FPGAs), and he is a leading expert in the areas of image and signal processing. He has authored more than 40 technical research papers. He is currently working on advanced sensor signal processing, algorithm development, and simulation for the Autonomous Landing and Hazard 
Avoidance Technology (ALHAT) project. The primary goal of the ALHAT project is to develop advanced technologies that will assist in safely landing crewed, cargo, and robotic vehicles on planetary bodies.

Bruce W. Barnes received a B.S. degree in Electrical Engineering from Old Dominion University in Norfolk, Virginia in 2001. He has been working for NASA Langley Research Center since 1987. He has been working on a number of laser remote sensing systems. These systems include the measurement of temperature, wind, $\mathrm{CO} 2$, vehicle motion and attitude, as well as hypersonic flow field profiling. His work currently supports the ALHAT Project on the development and implementation of data acquisition and control systems for the Doppler Lidar and Long Range Laser Altimeter. 